\title{
Development of post-COVID-19 cardiovascular events: an analysis of clinical features and risk factors from a single hospital retrospective study
}

\author{
Gianluca Cuomo ${ }^{1}$, Cinzia Puzzolante ${ }^{1}$, Vittorio ladisernia ${ }^{1,2}$, Antonella Santoro', \\ Marianna Menozzi ${ }^{1}$, Federica Carli¹, Margherita Digaetano' ${ }^{1}$, Gabriella Orlando', \\ Erica Franceschini ${ }^{1}$, Andrea Bedini ${ }^{1}$, Marianna Meschiari ${ }^{1}$, Lisa Manzini ${ }^{1}$, Luca Corradi' \\ Jovana Milic ${ }^{2}$, Vanni Borghi ${ }^{2}$, Lucio Brugioni ${ }^{3}$, Antonello Pietrangelo ${ }^{2,4}$, Enrico Clini2,5, \\ Massimo Girardis ${ }^{2,6}$, Giovanni Guaraldi1,2, Cristina Mussini ${ }^{1,2}$ \\ ${ }^{1}$ Infectious Diseases Clinic, Azienda Ospedaliero-Universitaria di Modena, Modena, Italy; \\ ${ }^{2}$ University of Modena and Reggio Emilia, Italy; \\ ${ }^{3}$ Internal Medicine and Critical Area Unit, Azienda Ospedaliero-Universitaria di Modena, Modena, Italy; \\ 4Internal Medicine Clinic, Azienda Ospedaliero-Universitaria di Modena, Modena, Italy; \\ ${ }^{5}$ Pneumology Clinic, Azienda Ospedaliero-Universitaria di Modena, Modena, Italy; \\ ${ }^{6}$ Intensive Care Unit, Azienda Ospedaliero-Universitaria di Modena, Modena, Italy
}

Article received 22 September, 2021; accepted 10 November, 2021

\section{SUMMARY}

Cardiovascular complications after a SARS-CoV-2 infection are a phenomenon of relevant scientific interest. The aim of this study was to analyze the onset of post-COVID-19 cardiovascular events in patients hospitalized in a tertiary care center.

This is a retrospective study conducted on patients hospitalized over a period of three months. The patients were older than 18 years of age and had a diagnosis of COVID-19 infection confirmed from a nasopharyngeal swab sample. Anamnestic and clinical-laboratory data were collected. Cardiovascular events at 30 days were defined as follows: arrhythmias, myocardial infarction, myocarditis, and pulmonary embolism. Univariate analysis (Student's t-test or Mann-Whitney U test, as appropriate) and multivariate analysis (multinomial logistic regression) were applied to the data.

A total of 394 patients were included; they were mostly males and had a median age of 65.5 years. Previous cardiovascular disease was present in $14.7 \%$ of patients. Oxygen therapy was required for $77.9 \%$, and $53 \%$ received anticoagulant therapy. The overall 30-day mortality was $20.3 \%$. A cardiovascular event developed in $15.7 \%$ of the subjects. These were mainly pulmonary embolism (9.4\%), followed by arrhythmias (3.3\%), myocardial infarction $(2.3 \%)$, and myocarditis $(0.8 \%)$. Patients who developed cardiovascular events upon univariate analysis were significantly older, with major comorbidities, a more compromised respiratory situation, and a higher mortality rate. Multivariate analysis revealed independent factors that were significantly associated with the development of cardiovascular events: hypertension, endotracheal intubation, and age older than 75 years.

In patients with COVID-19, the development of a cardiovascular event occurs quite frequently and is mainly seen in elderly subjects with comorbidities (especially hypertension) in the presence of a severe respiratory picture.

Keywords: COVID-19, cardiovascular diseases, SARSCoV-2.
Corresponding author

Gianluca Cuomo

E-mail: cuomo.gianluca@aou.mo.it

\section{INTRODUCTION}

evere acute respiratory syndrome coronavirus 22 (SARS-CoV-2) was identified as the virus responsible for a specific disease defined as coronavirus disease 2019 (COVID-19), which over the 
course of 2020 became a global pandemic. The key pathophysiological element of severe cases of the disease is a pattern of acute respiratory distress syndrome (ARDS) with alveolar lung damage, including alveolar hyaline membranes. Although in most cases COVID-19 symptoms are predominantly pulmonary, there are reports of signs of acute heart failure, arrhythmias, hypotension, tachycardia, and a high number of concomitant events, especially in patients admitted to intensive care unit [1]. The Italian and European Society of Cardiology has recommended caution, as physicians may place less emphasis on cardiological symptoms masked by more obvious respiratory symptoms $[2,3]$.

To date, the studies performed on the association between cardiovascular diseases and COVID-19 have been retrospective observational analyses, often from single centers. A meta-analysis of six studies including 1,527 patients with COVID-19 reported the rates of hypertension, cardiovascular and cerebrovascular disease, and diabetes to be $17.1 \%, 16.4 \%$, and $9.7 \%$, respectively [4]. Moreover, patients affected by these comorbidities required admission more frequently to intensive care units. In addition, in an extensive analysis by $\mathrm{Wu}$ et al., higher mortality rates were observed in patients with COVID-19 affected by cardiovascular disease $(10.5 \%)$, diabetes $(7.3 \%)$, and high blood pressure $(6.0 \%)$ than in those without these comorbidities (2.3\%) [5]. Some initial data on the Italian population seem to confirm the increased risk of mortality in subjects with these co-morbidities [6]. According to studies published to date, it appears that subjects with previous cardiovascular disease and cardiovascular risk factors may have a higher risk of COVID-19 as well as a poorer prognosis $[7,8]$. Concerning acute cardiac consequences of COVID-19, one of the first studies conducted in China on 41 patients with COVID-19 showed an increase of cardiac troponin at high sensitivity $(>28 \mathrm{pg} / \mathrm{ml})$ in five patients [9]. In another study conducted on 138 patients, 36 had severe symptoms and were treated in intensive care, and an increase in the concentration of cardiac markers of myocyte necrosis was reported (median creatine kinase concentration of 18 $\mathrm{U} / \mathrm{I}$ compared to $14 \mathrm{U} / \mathrm{I}$ ) [10]. These data suggest that patients with severe pulmonary symptoms often have complications affecting myocardial tissue [11].

High levels of serum troponin have been de- scribed in many patients affected by COVID-19, with significant differences in serum levels between deceased patients and survivors [12]. In a meta-analysis of four studies that included a total of 341 patients, the average cardiac troponin I levels were significantly higher in patients with severe COVID-19 disease than in patients with milder disease [13].

Recent studies have revealed that $19-27 \%$ of hospitalized patients with moderate and severe forms of COVID-19 develop acute myocardial damage, represented by an increase in troponin above the 99th percentile of the upper limit of reference (ultra-sensitive troponin I or troponin T) [14, 15]. Among patients with acute myocardial damage, $50-60 \%$ died, and in the group of patients with acute myocardial damage, mortality was significantly increased (compared to patients without acute myocardial damage), even among those who did not have a known history of cardiovascular disease [16]. In addition, several cases of acute myocarditis related to COVID-19 have been described [17, 18]. Arrhythmias have been described in numerous cases of patients with $\mathrm{CO}-$ VID-19. For example, the symptom "palpitations" was present as a presenting symptom in $7.3 \%$ of a cohort of 137 patients admitted to hospital with a diagnosis of COVID-19 [19]. In another cohort of patients hospitalized in China, cardiac arrhythmias were reported in $16.7 \%$ of cases and were much more frequent in patients admitted to intensive care [20]. Venous thromboembolism is another comorbidity to be considered. In fact, since these patients are hospitalized for a long time with the infection, and often in critical conditions, they are at high risk of developing venous thromboembolism [21].

The aim of this study was to analyze the incidence of cardiovascular events in patients hospitalized for SARS-CoV-2 infection.

\section{PATIENTS AND METHODS}

This was a retrospective, observational cohort study of prospectively collected data conducted in five hospital wards (Infectious Diseases, Pneumology, Intensive Care Unit, Internal Medicine, and Critical Area) of Modena University Hospital (Italy). The study population was adults $(\geq 18$ years) with COVID-19 confirmed by polymerase chain reaction (PCR) from a nasopharyngeal swab sample who were admitted to the centers 
between February 21, 2020 and May 31, 2020. All consecutive patients hospitalized for COVID-19 pneumonia documented on chest $X$-ray during the study period were considered eligible. The criterion for hospitalization was the presence of respiratory failure requiring any oxygen support. All subjects who survived had a 30-day follow-up after hospital discharge. Data on baseline signs, symptoms, comorbidities, treatments, outcomes, blood count, and biochemical and cardiac markers were collected. All data, including electrocardiograms (ECGs), were electronically recorded. All patients received standard of care treatment at the time of hospital admission according to the regional COVID-19 guidelines of Emilia Romagna [22]. Azithromycin $500 \mathrm{mg}$ per day, hydroxychloroquine (400 $\mathrm{mg} \times 2$ on days 1 and 2 and then $200 \mathrm{mg} \times 2$ from day 3 to day 6 ), and oxygen support to target an $\mathrm{SaO} 2$ reaching at least $90 \%$ were part of the standard treatment. Prophylaxis with low-molecular-weight heparin consisted of 4000 $\mathrm{U}$ once a day for weight under $100 \mathrm{~kg}$ and 6000 $\mathrm{U}$ once a day for weight over $100 \mathrm{~kg}$. Based on scientific evidence published during the study period, no patients received darunavir/cobicistat after March 18 [23]. Cardiovascular events were defined as follows:

1) acute myocardial infarction, defined by typical electrocardiographic alterations not previously known and troponin alteration above the 99th percentile of the reference values;

2) new onset of ECG documented arrhythmia, specifically atrial fibrillation, atrial flutter, and paroxysmal supraventricular tachycardia;

3) myocarditis, whose diagnosis was based on clinical and radiological criteria (echocardiogram and/or cardiac MRI) in accordance with European guidelines [24];

4) pulmonary thromboembolism, diagnosed on the clinical suspicion of the treating physician and always confirmed by computed tomography angiography.

\section{Statistical analyses}

Continuous variables were summarized as median and 95\% confidence interval. Categorical variables were summarized as absolute and relative frequencies. For descriptive analysis, continuous variables were compared using Student's t-test or the Mann-Whitney U test, as appropriate. Categorical variables were compared using the chi-square test or Fisher's exact test. $\mathrm{P}$-values less than 0.05 were considered statistically significant. Multinomial logistic regression was performed for the analysis of independent risk factors. All the factors that were significant to the descriptive analysis were included. The numerical variables that were significant were categorized on the basis of the median value. Analyses were performed using the Statistical Package for the Social Sciences (SPSS) version 26.0 (IBM Corp., Armonk, NY, USA). All procedures performed in the study were in accordance with the 1964 Declaration of Helsinki and its later amendments or comparable ethical standards and were approved by the institutional ethical committee.

\section{RESULTS}

During the observation period, 394 patients were enrolled. Table 1 presents the demographic, anamnestic, and clinical characteristics of the study population. Males accounted for $69.8 \%$ of the patients, and the median age was 65.5 years. Comorbidities were present in $35.7 \%$ of the patients, with the most represented condition being hypertension $(29.4 \%)$. A positive clinical history

Table 1 - General characteristics of the study population.

\begin{tabular}{|c|c|c|c|c|c|c|c|c|c|c|c|c|}
\hline \multicolumn{13}{|c|}{ Total Patients: 394} \\
\hline \multicolumn{2}{|c|}{$\begin{array}{c}\text { Anamnestic } \\
\text { data }\end{array}$} & $\begin{array}{c}\text { N/ } \\
\text { Median }\end{array}$ & $\begin{array}{l}95 \% \\
\mathrm{CI} / \%\end{array}$ & $\begin{array}{c}\text { Signs \& } \\
\text { symptoms }\end{array}$ & $\begin{array}{c}\text { N/ } \\
\text { Median }\end{array}$ & $\begin{array}{l}95 \% \\
\mathrm{CI} / \%\end{array}$ & $\begin{array}{l}\text { Chemical } \\
\text { parameters }\end{array}$ & Median & $\begin{array}{c}95 \% \\
\mathrm{CI}\end{array}$ & $\begin{array}{c}\text { Clinical } \\
\text { diary }\end{array}$ & $\begin{array}{c}\text { N/ } \\
\text { Median }\end{array}$ & $\begin{array}{l}95 \% \\
\text { CI } / \%\end{array}$ \\
\hline \multirow[t]{2}{*}{ Sex } & M & 275 & 69.8 & $\begin{array}{l}\text { Mean blood } \\
\text { pressure }\end{array}$ & $\begin{array}{c}95 \\
\mathrm{mmHg}\end{array}$ & $\begin{array}{l}95- \\
100\end{array}$ & $\begin{array}{c}\text { Lactates } \\
\mathrm{mg} / \mathrm{dL}\end{array}$ & 8 & $7-10$ & $\mathrm{P} / \mathrm{F}<250$ & 227 & 57.6 \\
\hline & F & 119 & 30.2 & Temperature & 37 & $37-38$ & $\begin{array}{c}\text { GPT_ALT } \\
\text { UI/L }\end{array}$ & 31 & $28-35$ & $\mathrm{P} / \mathrm{F}<150$ & 170 & 43.1 \\
\hline \multicolumn{2}{|l|}{ Age } & 65.5 & $63-69$ & Breathing rate & 22 & $18-23$ & $\begin{array}{c}\text { Creatinine } \\
\mathrm{mg} / \mathrm{dL}\end{array}$ & 0.81 & $\begin{array}{c}0.79- \\
0.86\end{array}$ & $\mathrm{P} / \mathrm{F}$ & 263 & $\begin{array}{l}250- \\
270\end{array}$ \\
\hline
\end{tabular}




\begin{tabular}{|c|c|c|c|c|c|c|c|c|c|c|c|}
\hline \multicolumn{12}{|c|}{ Total Patients: 394} \\
\hline $\begin{array}{c}\text { Anamnestic } \\
\text { data }\end{array}$ & $\begin{array}{c}\text { N/ } \\
\text { Median }\end{array}$ & $\begin{array}{l}95 \% \\
\mathrm{CI} / \%\end{array}$ & $\begin{array}{c}\text { Signs } \mathcal{E} \\
\text { symptoms }\end{array}$ & $\begin{array}{c}\text { N/ } \\
\text { Median }\end{array}$ & $\begin{array}{l}95 \% \\
\mathrm{CI} / \%\end{array}$ & $\begin{array}{l}\text { Chemical } \\
\text { parameters }\end{array}$ & Median & $\begin{array}{c}95 \% \\
\text { CI }\end{array}$ & $\begin{array}{c}\text { Clinical } \\
\text { diary }\end{array}$ & $\begin{array}{c}\text { N/ } \\
\text { Median }\end{array}$ & $\begin{array}{l}95 \% \\
\mathrm{CI} / \%\end{array}$ \\
\hline BMI & 26.7 & $\begin{array}{l}26.12- \\
27.78\end{array}$ & Dyspnea & 209 & 53 & $\begin{array}{c}\text { Hemoglobin } \\
\text { g/dL }\end{array}$ & 12.30 & $\begin{array}{c}12.20- \\
12.7\end{array}$ & Darunavir & 174 & 44.2 \\
\hline Comorbidity & 148 & 37.6 & Cough & 113 & 28.7 & $\begin{array}{l}\text { White blood } \\
\text { cells } \mathrm{n} / \mathrm{mmc}\end{array}$ & 6190 & $\begin{array}{c}5880- \\
6920\end{array}$ & $\begin{array}{c}\text { Immunological } \\
\text { therapy }\end{array}$ & 180 & 45.7 \\
\hline COPD & 22 & 5.6 & Conjunctivitis & 8 & 2.0 & $\begin{array}{l}\text { Platelets } \\
103 / \mathrm{mmc}\end{array}$ & 207 & $\begin{array}{l}193- \\
224\end{array}$ & Anakinra & 8 & 2.0 \\
\hline Diabetes & 45 & 11.4 & Rhinorrhea & 4 & 1.0 & LDH UI/L & 50 & $47-53$ & Tocilizumab & 172 & 43.7 \\
\hline Hypertension & 116 & 29.4 & $\begin{array}{l}\text { Pharyngo- } \\
\text { dynia }\end{array}$ & 11 & 2.8 & $\begin{array}{l}\text { Sodium } \\
\mathrm{mEq} / \mathrm{L}\end{array}$ & 137 & $\begin{array}{l}137- \\
138\end{array}$ & $\begin{array}{l}\text { Anticoagulant } \\
\text { therapy } \\
\text { (prophylaxis } \\
\text { at entry/ } \\
\text { already in Tp) }\end{array}$ & 209 & 53 \\
\hline $\begin{array}{l}\text { Previous } \\
\text { cardiovascular } \\
\text { disease } \\
\text { - Ischemic } \\
\text { cardiopathy } \\
\text { - Atrial } \\
\text { fibrillation } \\
\text { - Valvular disease } \\
\text { - Hearth failure } \\
\text { - PE } \\
\text { - Other }\end{array}$ & $\begin{array}{c}58 \\
21 \\
17 \\
4 \\
\\
2 \\
2 \\
12\end{array}$ & $\begin{array}{c}14.7 \\
5.3 \\
4.3 \\
\\
0.5 \\
\\
0.5 \\
0.5 \\
3\end{array}$ & Headache & 25 & 6.3 & $\begin{array}{l}\text { Potassium } \\
\mathrm{mEq} / \mathrm{L}\end{array}$ & 3.8 & $\begin{array}{l}3.8- \\
3.9\end{array}$ & $\begin{array}{l}\text { Oxygen } \\
\text { therapy }\end{array}$ & 307 & 77.9 \\
\hline $\begin{array}{l}\text { Chronic renal } \\
\text { failure }\end{array}$ & 21 & 5.3 & $\begin{array}{c}\text { Purulent } \\
\text { sputum }\end{array}$ & 7 & 1.8 & $\begin{array}{l}\text { Glycemia } \\
\mathrm{mg} / \mathrm{dL}\end{array}$ & 92 & $\begin{array}{l}86- \\
101\end{array}$ & NIV & 109 & 27.7 \\
\hline Cancer & 14 & 3.6 & $\begin{array}{l}\text { Not purulent } \\
\text { sputum }\end{array}$ & 15 & 3.8 & $\begin{array}{c}\text { PCR } \\
\mathrm{mg} / \mathrm{dL}\end{array}$ & 6.20 & $\begin{array}{c}6.10- \\
6.7\end{array}$ & Intubation & 72 & 18.3 \\
\hline $\begin{array}{l}\text { Other }(\mathrm{HIV}, \\
\text { hepatitis, } \\
\text { rheumatologic } \\
\text { diseases, } \\
\text { transplantation, } \\
\text { psychiatric } \\
\text { diseases) }\end{array}$ & 87 & 22.1 & Fatigue & 61 & 15.5 & $\begin{array}{c}\mathrm{PCT} \\
\mathrm{ng} / \mathrm{mL}\end{array}$ & 4.35 & $\begin{array}{c}0.20- \\
8.5\end{array}$ & $\begin{array}{c}\text { Death } \\
\text { Respiratory } \\
\text { Cardiovascular } \\
\text { Over-infection } \\
\text { Other }\end{array}$ & $\begin{array}{c}80 \\
77 \\
1 \\
1 \\
1\end{array}$ & $\begin{array}{c}20.3 \\
19.5 \\
0.2 \\
0.2 \\
0.2\end{array}$ \\
\hline $\begin{array}{l}\text { Hematologic } \\
\text { diseases }\end{array}$ & 9 & 2.3 & Hemoptysis & 5 & 1.3 & $\begin{array}{l}\text { Ferritin } \\
\mathrm{ng} / \mathrm{mL}\end{array}$ & 543 & $\begin{array}{l}392- \\
642\end{array}$ & & & \\
\hline Obesity & 41 & 10.4 & Diarrhea & 39 & 9.9 & $\begin{array}{c}\text { Troponin } \\
\text { ng/dL }\end{array}$ & 543 & $\begin{array}{l}392- \\
641\end{array}$ & & & \\
\hline Charlson score & 1 & $1-2$ & Myalgia & 17 & 4.3 & $\begin{array}{c}\mathrm{BNP} \\
\mathrm{ng} / \mathrm{dL}\end{array}$ & 69 & $\begin{array}{l}62- \\
273\end{array}$ & & & \\
\hline & & & Chills & 17 & 4.3 & SOFA score & 2 & $2-3$ & & & \\
\hline & & & $\begin{array}{c}\text { Tonsillar } \\
\text { edema }\end{array}$ & 1 & 0.3 & & & & & & \\
\hline & & & $\begin{array}{c}\text { Lympha- } \\
\text { denomegaly }\end{array}$ & 1 & 0.3 & & & & & & \\
\hline & & & $\begin{array}{c}\text { State of } \\
\text { consciousness } \\
\text { (altered) }\end{array}$ & 10 & 2.5 & & & & & & \\
\hline
\end{tabular}


for cardiovascular events was noted in $14.7 \%$ of the patients. The median Charlson score was 1 . In terms of signs and symptoms, $53 \%$ of the patients had dyspnea, and $28.7 \%$ had a cough. The median respiratory rate was 22 respirations per minute (other details in Table 1). Biohumoral results are presented in Table 1. The median SOFA score was 2 (more details in Table 1). Regarding COVID-19 therapies, $44.2 \%$ of the patients had therapy with darunavir/cobicistat. One-hundred and eighty $(45.7 \%)$ patients underwent immuno-

Table 2 - Development of cardiovascular events

\begin{tabular}{|l|c|c|}
\hline & No. & $\%$ \\
\hline Total cardiovascular events & 62 & 15.7 \\
\hline Arrhythmias & 13 & 3.3 \\
\hline Myocardial infarction & 9 & 2.3 \\
\hline Myocarditis & 3 & 0.8 \\
\hline Pulmonary embolism & 37 & 9.4 \\
\hline
\end{tabular}

logical therapy; $172(43.7 \%)$ received tocilizumab and eight $(2 \%)$ received anakinra. Two-hundred and nine $(53 \%)$ of the patients received prophylactic anticoagulant therapy; there were patients who were already being treated for previous diseases. Oxygen support was required for $77.9 \%$ of the patients; $27.7 \%$ underwent non-invasive ventilation (NIV), and $18.3 \%$ underwent endotracheal intubation. Overall mortality at 30 days was $20.3 \%$ (80 patients, 77 of whom died of respiratory causes).

During the follow-up after COVID-19, 62 patients (15.7\%) developed a cardiovascular event: 13 patients $(3.3 \%)$ had an arrhythmia, three patients $(0.8 \%)$ had myocarditis, nine patients $(2.3 \%)$ had an acute myocardial infarction, and 37 patients $(9.4 \%)$ had a pulmonary thromboembolism (Table 2).

The 62 patients with cardiovascular events (Table 3 ) were older ( 72.5 years vs $63, \mathrm{P}<0.001)$ and more frequently had chronic obstructive pulmonary disease (COPD) $(\mathrm{P}=0.006)$, diabetes $(\mathrm{P}=0.034)$, hyper-

Table 3 - Univariate analysis between patients who developed cardiovascular events and patients who did not.

\begin{tabular}{|c|c|c|c|c|c|}
\hline \multirow[t]{2}{*}{ Value } & \multicolumn{2}{|c|}{$\begin{array}{l}\text { Patients with cardiovascular } \\
\text { event development }(N=62)\end{array}$} & \multicolumn{2}{|c|}{$\begin{array}{c}\text { Patients with no cardiovascular } \\
\text { event development }(N=332)\end{array}$} & \multirow[t]{2}{*}{ P-Value } \\
\hline & Median/N & $I Q R / \%$ & Median/N & $I Q R / \%$ & \\
\hline \multicolumn{6}{|l|}{ Anamnestic data } \\
\hline Sex & 42 & 67.7 & 233 & 70.2 & 0.701 \\
\hline Age & 72.5 & $67-75$ & 63 & $61-67$ & $<0.001$ \\
\hline BMI & 26.12 & $25.47-31.14$ & 26.99 & $26.23-27.78$ & 0.346 \\
\hline Comorbidities & 31 & 50 & 117 & 35.2 & 0.08 \\
\hline COPD & 8 & 12.9 & 14 & 4.2 & 0.006 \\
\hline Diabetes & 11 & 17.7 & 34 & 10.2 & 0.034 \\
\hline Hypertension & 33 & 53.2 & 83 & 25 & $<0.001$ \\
\hline $\begin{array}{l}\text { Previous cardiovascular disease } \\
\text { - Ischemic cardiopathy } \\
\text { - Atrial fibrillation } \\
\text { - Valvular disease } \\
\text { - Hearth failure } \\
\text { - PE } \\
\text { - Other }\end{array}$ & $\begin{array}{c}19 \\
8 \\
7 \\
1 \\
1 \\
1 \\
1\end{array}$ & 30.6 & $\begin{array}{c}39 \\
13 \\
10 \\
3 \\
1 \\
1 \\
11\end{array}$ & 11.7 & $<0.001$ \\
\hline Chronic renal injury & 8 & 12.9 & 13 & 3.9 & 0.004 \\
\hline Neoplastic disease & 4 & 6.3 & 10 & 3.0 & 0.017 \\
\hline Others & 16 & 25.8 & 71 & 21.4 & 0.441 \\
\hline Hematological disease & 1 & 1.6 & 8 & 2.4 & 64 \\
\hline Obesity & 10 & 16.1 & 31 & 9.3 & 0.071 \\
\hline Charlson score & 2 & $1-3$ & 1 & $1-2$ & 0.299 \\
\hline
\end{tabular}


>> Continue

\begin{tabular}{|c|c|c|c|c|c|}
\hline \multirow[t]{2}{*}{ Value } & \multicolumn{2}{|c|}{$\begin{array}{l}\text { Patients with cardiovascular } \\
\text { event development }(N=62)\end{array}$} & \multicolumn{2}{|c|}{$\begin{array}{l}\text { Patients with no cardiovascular } \\
\text { event development }(N=332)\end{array}$} & \multirow[t]{2}{*}{ P-Value } \\
\hline & Median $/ \mathrm{N}$ & $I Q R / \%$ & Median $/ N$ & $I Q R / \%$ & \\
\hline \multicolumn{6}{|l|}{ Signs \& symptoms } \\
\hline Mean blood pressure & 95 & $90-105$ & 95 & $95-100$ & 0.432 \\
\hline Temperature & 37 & $37-38$ & 37 & $37-38$ & 0.110 \\
\hline Breathing rate & 23 & $20-26$ & 22 & $22-25$ & 0.394 \\
\hline Dyspnea & 40 & 64.5 & 169 & 50.9 & 0.049 \\
\hline Cough & 17 & 27.4 & 96 & 28.9 & 0.268 \\
\hline Conjunctivitis & 1 & 1.6 & 7 & 2.1 & 0.254 \\
\hline Rhinorrhea & 0 & 0.0 & 4 & 1.2 & 0.173 \\
\hline Pharyngodynia & 2 & 3.2 & 9 & 2.7 & 0.235 \\
\hline Headache & 2 & 3.2 & 23 & 6.9 & 0.103 \\
\hline Purulent sputum & 1 & 11.6 & 6 & 1.8 & 0.229 \\
\hline Not purulent sputum & 4 & 6.5 & 11 & 3.3 & 0.163 \\
\hline Fatigue & 12 & 19.4 & 49 & 14.8 & 0.164 \\
\hline Hemoptysis & 0 & 0.0 & 5 & 1.5 & 0.142 \\
\hline Diarrhea & 5 & 8.1 & 34 & 10.2 & 0.179 \\
\hline Myalgia & 3 & 4.8 & 14 & 4.2 & 0.235 \\
\hline Chills & 1 & 1.6 & 16 & 4.8 & 0.095 \\
\hline Tonsillar edema & 0 & 0.0 & 1 & 0.3 & 0.165 \\
\hline Lymphadenomegaly & 0 & 0.0 & 1 & 0.3 & 0.165 \\
\hline State of consciousness (altered) & 5 & 8.1 & 5 & 1.5 & 0.003 \\
\hline \multicolumn{6}{|l|}{ Clinical diary } \\
\hline $\mathrm{P} / \mathrm{F}<250 \mathrm{mmHg}$ & 42 & 67.7 & 185 & 55.7 & 0.079 \\
\hline $\mathrm{P} / \mathrm{F}<150 \mathrm{mmHg}$ & 36 & 58.1 & 133 & 40.1 & 0.009 \\
\hline $\mathrm{P} / \mathrm{F}$ Value & 176 & $97-263$ & 266 & $256-275$ & $<0.001$ \\
\hline Darunavir & 19 & 30.6 & 155 & 46.7 & 0.020 \\
\hline Immunological therapy & 33 & 53.2 & 147 & 44.3 & 0.194 \\
\hline Anakinra & 2 & 3.2 & 6 & 1.8 & \\
\hline Tocilizumab & 31 & 50.0 & 141 & 42.5 & \\
\hline $\begin{array}{l}\text { Anticoagulant } \\
\text { therapy (prophylaxis at entry/already on Tp) }\end{array}$ & 36 & 58.1 & 173 & 52.1 & 0.388 \\
\hline Oxygen therapy & 49 & 79.0 & 258 & 77.7 & 0.818 \\
\hline NIV & 28 & 45.2 & 81 & 24.4 & $<0.001$ \\
\hline Intubation & 22 & 35.5 & 50 & 15.1 & $<0.001$ \\
\hline $\begin{array}{l}\text { Death } \\
\text { - Respiratory } \\
\text { - Cardiovascular } \\
\text { - Over-infection } \\
\text { - Other }\end{array}$ & $\begin{array}{c}28 \\
27 \\
1 \\
0 \\
0\end{array}$ & $\begin{array}{c}45.2 \\
96.4 \\
3.6 \\
0.0 \\
0.0\end{array}$ & $\begin{array}{c}52 \\
50 \\
0 \\
1 \\
1\end{array}$ & $\begin{array}{c}15.7 \\
96.2 \\
0.0 \\
1.9 \\
1.9\end{array}$ & $<0.001$ \\
\hline
\end{tabular}




\begin{tabular}{|c|c|c|c|c|c|}
\hline \multirow[t]{2}{*}{ Value } & \multicolumn{2}{|c|}{$\begin{array}{c}\text { Patients with cardiovascular } \\
\text { event development }(N=62)\end{array}$} & \multicolumn{2}{|c|}{$\begin{array}{c}\text { Patients with no cardiovascular } \\
\text { event development }(N=332)\end{array}$} & \multirow[t]{2}{*}{$P$-Value } \\
\hline & Median $/ \mathrm{N}$ & $I Q R / \%$ & Median/N & $I Q R / \%$ & \\
\hline \multicolumn{6}{|l|}{ Chemical parameters } \\
\hline Lactates mg/dL & 12 & $11-18$ & 7 & $6-9$ & 0.015 \\
\hline GPT_ALT UI/L & 36 & $26-42$ & 30 & $26-34$ & 0.695 \\
\hline Creatinine $\mathrm{mg} / \mathrm{dL}$ & 0.87 & $0.77-0.83$ & 0.8 & $0.77-0.83$ & 0.124 \\
\hline Hemoglobin g/dL & 11.5 & $10.7-12.7$ & 12.3 & $12.2-12.7$ & 0.027 \\
\hline White blood cells $\mathrm{n} / \mathrm{mmc}$ & 7250 & $5600-8130$ & 6155 & $5800-6910$ & 0.819 \\
\hline Platelets $10^{3} / \mathrm{mmc}$ & 214 & $179-287$ & 203 & $189-224$ & 0.126 \\
\hline LDH UI/L & 42 & $38-64$ & 51 & $49-54$ & 0.533 \\
\hline Sodium $\mathrm{mEq} / \mathrm{L}$ & 138 & $137-140$ & 137 & $137-138$ & 0.288 \\
\hline Potassium mEq/L & 3.9 & $3.7-4.1$ & 3.8 & $3.8-3.9$ & 0.215 \\
\hline Glycemia mg/dL & 100 & $86-108$ & 91 & $86-101$ & 0.870 \\
\hline PCR mg/dL & 6.60 & $6.1-7.6$ & 6.10 & $5.8-6.5$ & 0.033 \\
\hline Ferritin ng/mL & 142.5 & $69-207$ & 579 & $434-848$ & 0.130 \\
\hline Troponin ng/dL & 31 & $12-35$ & 12 & $12-30$ & 0.042 \\
\hline BNP ng/dL & 273 & $259-508$ & 36 & $29-92$ & 0.003 \\
\hline SOFA score & 5 & $4-7$ & 2 & $2-3$ & $<0.001$ \\
\hline
\end{tabular}

Table 4 - Multivariate analysis (logistic regression): independent factors associated with cardiovascular event development.

\begin{tabular}{|c|c|c|c|}
\hline Factor & OR & $95 \% \mathrm{CI}$ & P-value \\
\hline Sex & 0.684 & $0.334-1.401$ & 0.299 \\
\hline COPD & 1.072 & $1.072-3.045$ & 0.907 \\
\hline Hypertension & 2.228 & $1.005-4.940$ & 0.048 \\
\hline Diabetes & 0.603 & $0.231-1.574$ & 0.301 \\
\hline Previous cardiovascular disease & 1.852 & $0.697-4.923$ & 0.217 \\
\hline Renal chronic failure & 1.334 & $0.391-4.545$ & 0.645 \\
\hline Neoplastic disease & 1.075 & $0.242-4.774$ & 0.924 \\
\hline Dyspnea & 1.143 & $0.516-2.529$ & 0.742 \\
\hline State of consciousness & 0.308 & $0.067-1.421$ & 0.131 \\
\hline $\mathrm{P} / \mathrm{F}<150 \mathrm{mmHg}$ & 0.826 & $0.349-1.958$ & 0.665 \\
\hline Darunavir & 0.501 & $0.249-1.008$ & 0.053 \\
\hline NIV & 1.238 & $0.569-2.693$ & 0.591 \\
\hline Intubation & 4.936 & $2.178-11.190$ & $<0.001$ \\
\hline Age $>75 y$ & 2.032 & $1.014-4.075$ & 0.046 \\
\hline SOFA score $>2$ & 1.638 & $0.805-3.333$ & 0.173 \\
\hline Lactate $>12 \mathrm{mg} / \mathrm{dl}$ & 1.310 & $0.565-3.039$ & 0.530 \\
\hline Hemoglobin >12 gr/dl & 1.042 & $0.545-1.991$ & 0.901 \\
\hline $\mathrm{PCR}>5 \mathrm{mg} / \mathrm{dl}$ & 1.223 & $0.588-2.542$ & 0.173 \\
\hline
\end{tabular}


tension $(\mathrm{P}<0.001)$, chronic renal disease $(\mathrm{P}=0.004)$, and cancer $(\mathrm{P}=0.017)$. In terms of symptoms, patients who developed a cardiovascular event had significantly more dyspnea $(64.5 \%$ vs $50.9 \%$, $\mathrm{P}=0.049$ ) and more often had an altered state of consciousness $(8.1 \%$ vs $1.5 \%, \mathrm{P}=0.003)$. Concerning treatments for patients with a cardiovascular event, fewer received darunavir/cobicistat therapy $(30.6 \%$ vs $46.7 \%, \mathrm{P}=0.020)$, and more underwent NIV $(45.2 \%$ vs $24.4 \%, \mathrm{P}<0.001)$ and endotracheal intubation $(35.5 \%$ vs $15.1 \%, \mathrm{P}=0.001)$. Mortality was higher in the group of patients who developed a cardiovascular event $(45.2 \%$ vs $15.7 \%, \mathrm{P}<0.001)$, but there were no significant differences in the cause of death. Concerning laboratory parameters, the two groups showed significant differences in the values of lactates $(\mathrm{P}=0.015)$, haemoglobin $(\mathrm{P}=0.027), \mathrm{PCR}(\mathrm{P}=0.033)$, troponin $(\mathrm{P}=0.042)$, and brain natriuretic peptide (BNP) $(\mathrm{P}=0.003)$. The median SOFA score was significantly worse in the group with cardiovascular events (5 vs $2, \mathrm{P}<0.001)$. Multivariate analysis factors independently associated with the onset of cardiovascular events were hypertension (OR 2.228, $\mathrm{P}=0.048$ ), endotracheal intubation (OR 4.936, $\mathrm{P}<0.001)$, and age over 75 years (OR 2.032, $\mathrm{P}=0.046$ ).

\section{DISCUSSION}

In this work, we observed an incidence of a cardiovascular event occurring 30 days after hospitalization of $15.7 \%$. They were especially common in elderly patients with comorbidities and a more compromised clinical respiratory condition. The demographic and clinical presentations of the patients are comparable to those reported in other studies [25]. More than half of the patients had a very compromised respiratory situation, defined by a $\mathrm{P} / \mathrm{F}$ ratio $<250$. This is probably due to the fact that the patients had a high median age and that the study population included patients who were hospitalized in intensive care wards. Interestingly, $44 \%$ of the patients were treated with darunavir/cobicistat. These were the first patients with COVID-19 treated in our hospital when data on the lack of efficacy of protease inhibitors had not yet been published [26]. Almost half of the patients underwent immunological therapy with tocilizumab. This is because our hospital, and in particular the Infectious Diseases Center, led the tocilizumab in patients with Severe CO-
VID-19 Pneumonia Study (TESEO) on the use of tocilizumab in the treatment of COVID-19, which showed good results in terms of survival, especially in patients with a compromised respiratory situation [27]. The observed mortality rate at 30 days was $20 \%$. This result is substantially in line with what reported in the literature, considering a recent analysis of in-hospital mortality rates that varied in relation to several factors, such as comorbidity, severity of the clinical setting, and need for endotracheal intubation [28].

With regard to the rationale behind the relationship between viral infection and cardiovascular complications, the American College of Cardiology referred to what is known about influenza. Experts agree that influenza increases risks in people with past cardiovascular disease (coronary heart disease or heart failure) and those at risk of cardiovascular events (diabetics, obese, and hypertensive individuals) [29]. This is mainly for three reasons: the severe inflammatory response that occurs following infection, with a consequent increase in acute coronary syndromes; myocardial depression/myocarditis, resulting in heart failure; and the risk of arrhythmia, which is probably associated with acute inflammation.

In this study, the development of cardiovascular events 30 days after hospitalization occurred in approximately $15 \%$ of the patients. It should be noted that this prevalence is lower than what is reported in the literature. For example, a recent meta-analysis observed an incidence of acute cardiac damage of $25 \%$, while myocardial infarction occurred in $2.5 \%$ of the patients in the present study [30]. Different countries have adopted different strategies, both in terms of hospitalization criteria and in testing for COVID-19. These factors may be responsible for selection bias and may have influenced the estimates of the impact of the virus on populations, both in terms of lethality and in the characteristics of the examined populations. It is therefore likely that the incidence of cardiac manifestations may be high in patients with COVID-19. This may be understood to be not only about the risk of plaque instability linked to inflammation but also in terms of hypoxemia, potentially leading to a mismatch between supply and demand with the development of type II myocardial infarction, especially in the context of severe infection and ARDS. Furthermore, the severe inflammatory response and hemodynamic 
changes occurring during severe forms of COVID-19 could contribute to the instability of atherosclerotic plaques in predisposed individuals and thus to the development of acute coronary syndromes (type I myocardial infarction) [31].

Arrhythmias occurred in 3.3\% of the patients in this study, which is once again a lower percentage than what is reported in the literature, where the percentages vary between $7.5 \%$ and $16.7 \%[32,33]$. In all the studies mentioned, there does not seem to be a direct correlation between COVID-19 and cardiac arrhythmias; it seems to occur mainly due to systemic illness. However, it is relevant in the interpretation of the arrhythmia data that almost all of the patients in this study were treated with a combination of azithromycin plus hydroxychloroquine. These drugs are known to expose the patient to a risk of potentially fatal ventricular arrhythmias due to QT elongation [34]. With regard to the onset of myocarditis as a complication or as a clinical manifestation of infection with SARS-CoV-2, the literature is mainly based on case reports, and the data present many biases in relation to the difficult diagnosis and symptomatology, which is not easily distinguishable from the others typical of COVID-19 [35]. The onset of pulmonary thromboembolism or arterial and venous embolism in general has been widely reported in the literature, with data ranging from $7.7 \%$ to $14.7 \%$ to $23.4 \%$; this phenomenon is in relation to the admission department and the intensity of care [36-38]. The higher incidence of pulmonary embolism in the most critical patients is a fact already highlighted in the literature that has been confirmed by this study. Pulmonary embolism in COVID-19 patients is probably related to higher procoagulant activity linked to the increased state of inflammation $[39,40]$.

Overall, the development of cardiovascular events observed in this study is generally lower than what is reported in the literature. This is probably related to the high percentage of patients in this study (more than half) undergoing anticoagulant prophylaxis immediately after hospitalization. At the present time, scientific evidence suggests prophylaxis against thromboembolism with anticoagulants for all patients hospitalized with $\mathrm{CO}$ VID-19 [41].

Patients who developed a cardiovascular event had a history of major comorbidities, in particular COPD, diabetes, renal failure, previous cardiovas- cular events, and neoplastic diseases. These data seem to be consistent with what we already know about the general population in which the cardiovascular risk is higher in people with co-morbidities [42-44]. The onset of an additional stressful element such as COVID-19, with its cytokine storm and state of hypercoagulability, may have favored the development of a cardiovascular event in those subjects with a favorable substrate, which is represented by the known risk factors. To support this argument in our work, the patients who developed a cardiovascular event had a poorer basal respiratory situation, represented by a low $\mathrm{P} / \mathrm{F}$ ratio, increased use of NIV, and orotracheal intubation. Significantly more patients who developed a cardiovascular event died. The causes of death did not vary between the two groups, so the higher mortality in the cardiovascular group is probably due to the higher number of comorbidities and severity of COVID-19. It is known from the literature that underlying diseases are a risk factor for a severe trend of COVID-19 [45].

Apparently, fewer patients who developed a cardiovascular event received darunavir. This data is affected by a possible bias; in fact, as highlighted in the methods section, patients did not receive darunavir after March 18 due to a change in the therapeutic protocol. It is known that darunavir/ cobicistat has many pharmacological interactions, some of which can have a positive or negative impact on the development of cardiovascular events, especially arrhythmias [46]. Independently associated risk factors in the cardiovascular group were hypertension, advanced age, and endotracheal intubation. Age and hypertension have been extensively studied in the literature as cardiovascular risk factors [47, 48]. Intubation is also an independent risk factor. We know that in the general population, cardiovascular risk is increased in elderly patients with hypertension who are intubated for surgery and that this risk is increased especially in the first days after intubation [49]. In our opinion, orotracheal intubation is a proxy for the severity of SARS-CoV-2 disease, and, as already widely demonstrated, the most critical COVID-19 patients are at greater risk of mortality and severe events, including the development of cardiovascular events [50]. Thus, patients who developed a cardiovascular event were those who had greater respiratory and functional involvement. 
One important limitation of this study is that it is a retrospective study. Also, the analysis of the development of cardiovascular events was limited to 30 days due to lack of follow-up data. Another limitation is that cardiogenic shock, heart failure, and newly diagnosed valvulopathy were not considered among cardiovascular events.

In conclusion, we observed that post-COVID-19 cardiovascular complications are more frequent in elderly patients with hypertension and with more severe courses of COVID-19, as represented by the need for orotracheal intubation. The state of hyperinflammation and increased coagulation seems to favor the development of cardiovascular events in subjects already predisposed due to age and co-morbidity.

\section{Funding}

This study did not receive any specific grant from any funding agency in the public, commercial, or not-for-profit sectors.

\section{Ethics approval and consent to participate}

The study was reviewed and approved by the University of Modena e Reggio Emilia local institutional Ethical Committee (Comitato Etico Provinciale di Modena). The study was conducted in accordance with the provisions of the Declaration of Helsinki and Good Clinical Practice guidelines. All involved persons gave their informed verbal witnessed consent. Data were collected anonymously.

\section{Competing interests}

The authors declare that they have no competing interests.

\section{Authors' contributions}

Cuomo G participated in data collection, performed the statistical analysis, and wrote the manuscript. Puzzolante $C$ and Iadisernia V participated in the design of the study and the database application and helped draft the manuscript. Santoro A, Menozzi M, Carli F, Digaetano M, Orlando G, Franceschini E, Bedini A, Meschiari M, Manzini L, Corradi L, Milic J, Borghi V, Brugioni L, Pietrangelo A, Clini E, and Girardis M participated in data collection and patient recruitment. Guaraldi $G$ and Mussini $C$ were substantial contributors to the conception and design of the study, interpretation of data, critical revision of the manuscript, and final approval of the version to be published. All authors read and approved the final manuscript.

\section{Acknowledgements}

We would like to express our special thanks of gratitude to: Raimondi A, Rogati C, Ciusa G, Tutone M, Bacca E, Dolci G, Franceschi G, Faltoni M, Volpi S, Burastero G, Yaacoub D, Castaniere I, Tonelli R, Tabbì L, Fantini R, Gozzi F, Angrisani $D$, Vacchi $C$, for their unceasing efforts to care for COVID-19 patients.

\section{REFERENCES}

[1] Zheng YY, Ma YT, Zhang JY, Xie X. COVID-19 and the cardiovascular system. Nat Rev Cardiol. 2020; 17 (5), 259-60.

[2] Società Italiana di Cardiologia. Guida clinica Covid-19 per cardiologi. https://www.sicardiologia.it/ public/Documento-SIC-COVID-19.pdf

[3] Anker SD, Butler J, Khan MS, et al. Conducting clinical trials in heart failure during (and after) the COVID-19 pandemic: an Expert Consensus Position Paper from the Heart Failure Association (HFA) of the European Society of Cardiology (ESC). Eur Heart J. 2020; 41 (22), 2109-17.

[4] Li B, Yang J, Zhao F, et al. Prevalence and impact of cardiovascular metabolic diseases on COVID-19 in China. Clin Res Cardiol. 2020; 109 (5), 531-8.

[5] Wu Z, McGoogan JM. Characteristics of and Important Lessons from the Coronavirus Disease 2019 (COVID-19) Outbreak in China: Summary of a Report of 72314 Cases From the Chinese Center for Disease Control and Prevention. JAMA. 2020; 323 (13), 1239-42.

[6] Porcheddu R, Serra C, Kelvin D, Kelvin N, Rubino S. Similarity in Case Fatality Rates (CFR) of COVID-19/ SARS-COV-2 in Italy and China. I Infect Dev Ctries. 2020; 14 (2), 125-8.

[7] Zhou F, Yu T, Du R, et al. Clinical course and risk factors for mortality of adult inpatients with COVID-19 in Wuhan, China: a retrospective cohort study. Lancet. 2020; 395 (10229), 1054-62.

[8] Mai F, Del Pinto R, Ferri C. COVID-19 and cardiovascular diseases. J Cardiol. 2020; 76, 453-8.

[9] Huang C, Wang Y, Li X, et al. Clinical features of patients infected with 2019 novel coronavirus in Wuhan, China. Lancet. 2020; 395 (10223), 497-506.

[10] Ruan Q, Yang K, Wang W, Jiang L, Song J. Clinical predictors of mortality due to COVID-19 based on an analysis of data of 150 patients from Wuhan, China. Intensive Care Med. 2020; 46 (5), 846-8.

[11] Alali AH, Smaisem MS, Alsheikh AM, et al. Myocardial injuries among patients with COVID-19: a systematic review. Infez Med. 2021; 29, 345-54 
[12] Yang $X, Y u ~ Y, X u ~ J$, et al. Clinical course and outcomes of critically ill patients with SARS-CoV-2 pneumonia in Wuhan, China: a single-centered, retrospective, observational study. Lancet Respir Med. 2020; 8 (5), 475-81.

[13] Lippi G, Lavie CJ, Sanchis-Gomar F. Cardiac troponin I in patients with coronavirus disease 2019 (COVID-19): Evidence from a meta-analysis. Prog Cardiovasc Dis. 2020; 63 (3), 390-1.

[14] Chen T, Wu D, Chen H, et al. Clinical characteristics of 113 deceased patients with coronavirus disease 2019: retrospective study. BMJ. 2020; 368, m1091.

[15] Shi S, Qin M, Shen B, et al. Association of Cardiac Injury with Mortality in Hospitalized Patients With COVID-19 in Wuhan, China. JAMA Cardiol. 2020; 5 (7), 802-10.

[16] Guo T, Fan Y, Chen M, et al. Cardiovascular Implications of Fatal Outcomes of Patients with Coronavirus Disease 2019 (COVID-19). JAMA Cardiol. 2020; 5 (7), 811-8.

[17] Hu H, Ma F, Wei X, Fang Y. Coronavirus fulminant myocarditis treated with glucocorticoid and human immunoglobulin. Eur Heart J. 2021; 42 (2), 206.

[18] Cuomo G, Menozzi M, Carli F, et al. Acute myocarditis as the main clinical manifestation of SARS-CoV 2 infection. Infect Dis Rep. 2020; 12 (2), 8609.

[19] Liu K, Fang YY, Deng Y, et al. Clinical characteristics of novel coronavirus cases in tertiary hospitals in Hubei Province. Chin Med J (Engl). 2020; 133 (9), 1025-31.

[20] Wang D, Hu B, Hu C, et al. Clinical Characteristics of 138 Hospitalized Patients With 2019 Novel Coronavirus-Infected Pneumonia in Wuhan, China. JAMA. 2020; 323 (11), 1061-9.

[21] Vacchi C, Meschiari M, Milic J, et al. COVID19-associated vasculitis and thrombotic complications: from pathological findings to multidisciplinary discussion. Rheumatology (Oxford). 2020; 59 (12), e147-e150.

[22] Indirizzi terapeutici della Regione E-R per il trattamento della infezione da SARS-CoV2 (COVID-19. Available on: https://salute.regione.emilia-romagna.it/farmaci/covid-19/indirizzi-terapeutici-covid19-rer.pdf.

[23] Cao B, Wang Y, Wen D, et al. A trial of lopinavirritonavir in adults hospitalized with Severe Covid-19. N Engl J Med. 2020; 382 (19), 1787-99.

[24] Caforio AL, Pankuweit S, Arbustini E, et al. Current state of knowledge on aetiology, diagnosis, management, and therapy of myocarditis: a position statement of the European Society of Cardiology Working Group on Myocardial and Pericardial Diseases. Eur Heart J. 2013; 34 (33), 2636-48.

[25] Macera M, De Angelis G, Sagnelli C, Coppola N, Vanvitelli Covid-Group. Clinical Presentation of COVID-19: Case Series and Review of the Literature. Int J Environ Res Public Health. 2020; 17 (14), 5062.

[26] Chen J, Xia L, Liu L, et al. Antiviral Activity and
Safety of Darunavir/Cobicistat for the Treatment of COVID-19. Open Forum Infect Dis. 2020; 7 (7), ofaa241. [27] Guaraldi G, Meschiari M, Cozzi-Lepri A, et al. Tocilizumab in patients with severe COVID-19: a retrospective cohort study. Lancet Rheumatol. 2020; 2 (8), e474-e484. [28] Weiss P, Murdoch DR. Clinical course and mortality risk of severe COVID-19. Lancet. 2020; 395 (10229), 1014-5.

[29] American College Cardiology. Coronavirus Disease 2019 (COVID-19) Provides Potent Reminder of the Risk of Infectious Agents. 2020. https://www.acc. org/latest-in-cardiology/articles/2020/03/01/08/42/ feature-coronavirus-disease-2019-covid-19-providespotent-reminder-of-the-risk-of-infectious-agents.

[30] Momtazmanesh S, Shobeiri P, Hanaei S, MahmoudElsayed H, Dalvi B, Malakan Rad E. Cardiovascular disease in COVID-19: a systematic review and metaanalysis of 10,898 patients and proposal of a triage risk stratification tool. Egypt Heart J. 2020; 72 (1), 41.

[31] Guzik TJ, Mohiddin SA, Dimarco A, et al. COVID-19 and the cardiovascular system: implications for risk assessment, diagnosis, and treatment options. Cardiovasc Res. 2020; 116 (10), 1666-87.

[32] Bhatla A, Mayer MM, Adusumalli S, et al. COVID-19 and cardiac arrhythmias. Heart Rhythm. 2020; 17 (9), 1439-44.

[33] Kochi AN, Tagliari AP, Forleo GB, Fassini GM, Tondo C. Cardiac and arrhythmic complications in patients with COVID-19. J Cardiovasc Electrophysiol. 2020; 31 (5), 1003-8.

[34] Saleh M, Gabriels J, Chang D, et al. Effect of Chloroquine, Hydroxychloroquine, and Azithromycin on the Corrected QT Interval in Patients With SARS-CoV-2 Infection. Circ Arrhythm Electrophysiol. 2020; 13 (6), e008662. [35] Siripanthong B, Nazarian S, Muser D, et al. Recognizing COVID-19-related myocarditis: The possible pathophysiology and proposed guideline for diagnosis and management. Heart Rhythm. 2020;17 (9), 1463-71.

[36] Danzi GB, Loffi M, Galeazzi G, Gherbesi E. Acute pulmonary embolism and COVID-19 pneumonia: a random association? Eur Heart J. 2020; 41 (19), 1858.

[37] Lodigiani C, Iapichino G, Carenzo L, et al. Venous and arterial thromboembolic complications in COVID-19 patients admitted to an academic hospital in Milan, Italy. Thromb Res. 2020; 191, 9-14.

[38] Roncon L, Zuin M, Barco S, et al. Incidence of acute pulmonary embolism in COVID-19 patients: Systematic review and meta-analysis. Eur J Intern Med. 2020; 82, 29-37.

[39] Spiezia L, Boscolo A, Poletto F, et al. COVID-19-Related Severe Hypercoagulability in Patients Admitted to Intensive Care Unit for Acute Respiratory Failure. Thromb Haemost. 2020; 120 (6), 998-1000.

[40] Montazerin SM, Majafi H, Shojaei F, et al. COVID19-associated coagulopathy: a concise review on pathogenesis and clinical implications. Infez Med. 2021; 29, 1-7. [41] Barnes GD, Burnett A, Allen A, et al. Thrombo- 
embolism and anticoagulant therapy during the $\mathrm{CO}$ VID-19 pandemic: interim clinical guidance from the anticoagulation forum. J Thromb Thrombolysis. 2020; 50 (1), 72-81.

[42] Tzoulaki I, Elliott P, Kontis V, Ezzati M. Worldwide Exposures to Cardiovascular Risk Factors and Associated Health Effects: Current Knowledge and Data Gaps. Circulation. 2016; 133 (23), 2314-33.

[43] Rabe KF, Hurst JR, Suissa S. Cardiovascular disease and COPD: dangerous liaisons? Eur Respir Rev. 2018; 27 (149), 180057.

[44] Hoeger CW, Hayek SS. Role of Cardiovascular Biomarkers in the Risk Stratification, Monitoring, and Management of Patients with Cancer. Cardiol Clin. 2019; 37 (4), 505-23.

[45] Yang J, Zheng Y, Gou X, et al. Prevalence of comorbidities and its effects in patients infected with SARS-
CoV-2: a systematic review and meta-analysis. Int J Infect Dis. 2020; 94, 91-5.

[46] Navarro J, Curran A. Profile of once-daily darunavir/cobicistat fixed-dose combination for the treatment of HIV / AIDS. HIV AIDS (Auckl). 2016; 8, 175-82.

[47] Buford TW. Hypertension and aging. Ageing Res Rev. 2016; 26, 96-111.

[48] Oliveros E, Patel H, Kyung S, et al. Hypertension in older adults: Assessment, management, and challenges. Clin Cardiol. 2020; 43 (2), 99-107.

[49] Zhang J, Wang Z, Ji Y, et al. Risk analysis of cardiovascular events in elderly hypertensive patients with different risk stratification during the perioperative period. Zhonghua Yi Xue Za Zhi. 2015; 95 (28), 2258-63.

[50] Murthy S, Gomersall CD, Fowler RA. Care for Critically Ill Patients With COVID-19. JAMA. 2020; 323 (15), 1499-500. 